\title{
Effect of Storage Conditions on Rate of Color Degradation of Paprika based Products
}

\author{
Ramya Addala, Mihir Vasavada*, Jane Dong and Siva Subramanian
}

Olam Spices and Vegetable Ingredients, Fresno, CA, USA

\begin{abstract}
The purpose of this study was to determine the effect of storage conditions on rate of color degradation of paprika based products. The study was conducted on 7 paprika, 3 chili pepper and 2 chili powder products commercially produced at Olam Spices and Vegetable Ingredients. All products were collected from the manufacturing location at Las Cruces, NM and stored in ziploc bags for up to 6 months at 4 different storage conditions, namely: $35^{\circ} \mathrm{C} / 80 \% \mathrm{RH}$, room temperature $\sim 22^{\circ} \mathrm{C} / 45 \% \mathrm{RH}$, refrigerated temperature at $7^{\circ} \mathrm{C}$ and frozen temperature at $-8^{\circ} \mathrm{C}$. Representative samples were collected at Time $0,2,4,6,8,12$, 16, 20 and 24 weeks from each storage condition and analyzed for moisture and water activity (Aw), extractable color (ASTA) and surface color (Hunter L, a, b). Results showed that samples with ethoxyquin demonstrated a significantly lower extractable color loss than samples without ethoxyquin. Further, samples that had been irradiated demonstrated a significantly higher surface color loss than samples that had not been irradiated. Samples at high temperature - humidity storage degraded significantly rapidly compared to samples stored in room conditions. Refrigerated and freezer stored samples showed minimal extractable and visual color loss. This study provides significant information on storage stability of paprika based products during long term storage.
\end{abstract}

Keywords: Extractable color; Irradiation; Paprika

Abbreviations: ASTA: American Spice Trade Association; PACI : Paprika Color Index

\section{Introduction}

Dehydrated paprika (Capsicum annuum) based ground spice products can be divided into 3 major categories, namely: ground paprika, chili peppers and chili powders. These products are used in food applications throughout the world as natural food colorants or seasoning agents, due to their attractive color, unique sweet taste, flavor and pungency. Dehydrated paprika is mainly used for their extractable color (ASTA) and contains high ASTA paprika blended with ethoxyquin as an antioxidant to maintain desired ASTA levels. Chili peppers are mainly used for taste and flavor and mild to moderate heat and usually contain dehydrated paprika blended with ground cayenne red peppers or hot seed for providing heat and pungency. Chili powders are mainly used for direct applications on food, color and mild to moderate heat and usually contain chili peppers with added salt and blended with different dehydrated spices depending on the formulation.

The quality parameters of the stored dehydrated capsicum based products that are important to spice formulators are, in addition to microbiological status and absence of insect infestation, the retention of characteristic color, characteristic aroma, and content of capsaicinoids (pungency) [1,2]. Many factors such as cultivar, temperature of drying, oxygen/air atmosphere, temperature, and moisture content during storage affect the retention of color [3]. Typically products are stored and used between 6 months to 1 year from the date of manufacturing. Paprika being a seasonal raw material is dehydrated, ground and used in formulations typically in a 1 year cycle.

The red color intensity is considered the most important physical attribute for paprika. The red varieties of paprika are very rich sources of carotenoids, particularly capsanthin and capsorubin containing one and two keto groups, respectively. Qualitative and quantitative distribution of carotenoids in red paprika reveal that the red carotenoids (capsanthin, capsorubin, and cryptocapsin) are formed from the appropriate 5,6-epoxycarotenoids (antheraxanthin, violaxanthin, and cryptoxanthin 5,6-epoxide) [4]. In the traditional paprika dehydration processes, due to a prolonged exposure to heat, light, and oxygen, a $20-53 \%$ loss of the initial carotenoids and thereby the color of paprika has been reported [5]. Non-enzymatic browning is another cause of paprika color degradation. It has been found that the water activity and temperature had a significant effect on the non-enzymatic browning rate of dried red peppers during their storage [6]. Paprika is high in content of reducing sugars and amino acids so it is a good medium for Maillard reactions during its processing and storage of its products $[7,8]$.

Ethoxyquin is used in the spice industry as an antioxidant to prevent carotenoid loss during postharvest handling and has been used since Lease and Lease and Chen and Gutmanis proved its effectiveness for color retention in stored dehydrated paprika $[9,10]$. The council of European communities developed directives regarding authorization of food additives throughout the community and ethoxyquin was not listed as an approved additive [11]. FDA has reported no scientific or medical evidence that ethoxyquin used at approved levels is injurious to human or animal health [12]. Paprika products in the US generally use ethoxyquin for maintaining color stability during storage.

Irradiation treatments have been used to reduce the microbiological load of dehydrated paprika and the effect of irradiation on color loss of paprika is unclear. Studies on red paprika with irradiation doses between 0 to $12.5 \mathrm{kGy}$ showed no significant differences between the color properties of irradiated and non-irradiated samples [13]. However, another study suggested that higher irradiation doses and a

*Corresonding author: Mihir Vasavada, Olam International, Olam Spices and Vegetable Ingredients, 205 E, River Park Circle, Ste 310, Fresno, CA, USA, Tel: 559256 6236; E-mail: mihir.vasavada@olamnet.com

Received December 08, 2014; Accepted February 16, 2015; Published February 23, 2015

Citation: Addala R, Vasavada M, Dong J, Subramanian S (2015) Effect of Storage Conditions on Rate of Color Degradation of Paprika based Products. J Food Process Technol 6: 423. doi:10.4172/2157-7110.1000423

Copyright: (C) 2015 Addala R, et al. This is an open-access article distributed under the terms of the Creative Commons Attribution License, which permits unrestricted use, distribution, and reproduction in any medium, provided the original author and source are credited. 
longer storage period, resulted in a significant $(\mathrm{p}<0.01)$ reduction of all the carotenoids, except capsorubin [14]

Most studies conducted on dehydrated paprika based products have been conducted on different species of paprika and measuring the effect of heating parameters, microbial reduction treatments, storage conditions or added antioxidants on the color characteristics of paprika products. This study is significant because we have used products from a commercial facility and analyzed the effect of storage conditions on color and other parameters on these samples. This study would provide significant information on the color loss information on commercial paprika samples during long term storage. This information could be useful for specifying storage conditions and shelf life which can also be used for shipping products across different continents.

\section{Materials and Methods}

\section{Sample preparation and storage}

Twelve different product samples from 3 main product categories: 7 paprika, 3 peppers and 2 powders; were collected from our Las Cruces manufacturing plant and evaluated in this study. Approximately $6 \mathrm{lb}$ of each product sample was collected in polyethylene bags and sent to Olam's Innovation and Quality center at Fresno, California. Before beginning the shelf life study, each $6 \mathrm{lb}$ sample bag was opened and the product was divided into 4 polyethylene ziploc bags $(\sim 1.5 \mathrm{lb}$ in each bag) for each product sample. Each individual ziploc bag was labeled and placed into another larger polyethylene bag, to provide dual layer protection simulating an inner and outer package used for commercial products. The larger bags were tied and placed in 4 different storage conditions ( 1 bag in each condition) for each product sample type. Ultimately $\sim 1.5 \mathrm{lb}$ of each product sample was stored in each storage condition. The storage conditions for this study included freezer temperature $\left(-8^{\circ} \mathrm{C}\right.$ and $\left.45 \% \mathrm{RH}\right)$, refrigerator temperature $\left(7^{\circ} \mathrm{C}\right.$ and $55 \% \mathrm{RH})$, room temperature $\left(22^{\circ} \mathrm{C}\right.$ and $\left.45 \% \mathrm{RH}\right)$ and elevated temperature $\left(35^{\circ} \mathrm{C}\right.$ and $\left.80 \% \mathrm{RH}\right)$.

\section{Sample details}

The main ingredients and treatment details for all the paprika, chili pepper and chili powder samples are listed in Table 1 . The 7 paprika samples contained blends of different dehydrated paprika with different particle sizes and color, to meet our specifications. All 7 paprika products contained silicon dioxide as a flowing agent and all paprika samples except samples 2 and 6 contained ethoxyquin to preserve the red color. We specifically selected 2 samples of paprika without ethoxyquin, at different ASTA levels, to study the effect of

\begin{tabular}{|c|c|c|c|}
\hline Sample ID & Ingredients & Ethoxyquin & Irradiation \\
\hline Paprika 1 & Paprika, $\mathrm{SiO}_{2}$ & Yes & No \\
\hline Paprika 2 & Paprika, $\mathrm{SiO}_{2}$ & No & No \\
\hline Paprika 3 & Paprika, $\mathrm{SiO}_{2}$ & Yes & Yes \\
\hline Paprika 4 & Paprika, $\mathrm{SiO}_{2}$ & Yes & No \\
\hline Paprika 5 & Paprika, $\mathrm{SiO}_{2}$ & Yes & No \\
\hline Paprika 6 & Paprika, $\mathrm{SiO}_{2}$ & No & No \\
\hline Paprika 7 & Paprika, $\mathrm{SiO}_{2}$ & Yes & No \\
\hline Pepper 1 & Paprika, ground red pepper, $\mathrm{SiO}_{2}$ & Yes & No \\
\hline Pepper 2 & Paprika, ground red pepper, $\mathrm{SiO}_{2}$ & Yes & Yes \\
\hline Pepper 3 & Paprika, ground red pepper, $\mathrm{SiO}_{2}$ & Yes & No \\
\hline Powder 1 & Paprika, ground red pepper, spice blend, $\mathrm{SiO}_{2}$ & Yes & No \\
\hline Powder 2 & Paprika, ground red pepper, spice blend, $\mathrm{SiO}_{2}$ & No & No \\
\hline
\end{tabular}

Table 1: Ingredients in paprika, chili pepper and chili powder blends used in shelf life study. ethoxyquin on preserving ASTA color. Paprika sample 3 was subjected to irradiation treatment of $12-15 \mathrm{kGy}$ for microbial reduction purpose. The U.S. Food and Drug Administration irradiation dose limits for spices and seasonings is $30 \mathrm{kGy}$ [15]. The 3 chili pepper samples contained a combination of paprika and ground red pepper (for heat) in different proportions, to meet our specifications. All chili pepper samples contained silicon dioxide as a flowing agent and ethoxyquin for color preservation. Chili pepper 2 was also subjected to irradiation treatment of $12-15 \mathrm{kGy}$ for microbial reduction purpose. The 2 chili powder samples contained paprika, ground red pepper and spices (for flavor) in different proportions, to meet our specifications. Chili powder 1 contained ethoxyquin for color preservation whereas chili powder 2 did not contain ethoxyquin. None of the chili powder samples were irradiated for microbial reduction.

\section{Testing protocol}

All the products were tested for 4 parameters - moisture, water activity, extractable color (ASTA) and surface color (HUNTER) at each time point. Initially, the samples were tested for all the four parameters before they were placed in the respective storage conditions and the results were recorded as Day ' 0 ' data, considered as the initial data for the study. During the shelf life study, $\sim 40$ g sample each was collected for each of the 12 product samples from all 4 storage conditions and analyzed for the above parameters; throughout the study for 24 weeks. Hence, overall at each time point during the study, a total of 48 samples were analyzed, in duplicate, for all 4 testing parameters. For the first 8 weeks the samples were analyzed every 2 weeks and thereafter they were analyzed every 4 weeks up to 24 weeks. All the results were reported as the average of the duplicate readings.

\section{Moisture}

Moisture content for the samples was measured following ASTA 2.1 using vacuum oven method [16]. The samples were weighed $(2.0 \mathrm{~g}$ $3.0 \mathrm{~g}$ ) and placed in the oven (Thermo Fisher Scientific Lindberg / Blue M Vacuum Ovens VO914, Thermo Fisher Scientific Inc., Asheville, NC, www.thermoscientific.com) which was pre-heated to $70^{\circ} \mathrm{C}$ and vacuum of 2 - 4 in $\mathrm{Hg}$ units. The samples were dried for $6 \mathrm{~h}$ and then were cooled to ambient temperature in a desiccator. The samples were then weighed to calculate the $\%$ moisture. The average of duplicate readings was used for each sample in each condition at each time point. The average of duplicate readings was used for each sample in each condition at each time point.

\section{$\%$ Moisture $=[($ initial wt. - final wt. $) /$ initial wt. $]$ X 100}

\section{Water activity (Aw)}

The water activity for the samples was measured following ASTA 6.0 method using Aqua lab 4TE (Aqua lab by Decagon - Decagon Devices Inc., Pullman, WA, (www.decagon.com) [17]. The samples were measured before and during storage similar to other analyses mentioned earlier. The average of duplicate readings was used for each sample in each condition at each time point.

\section{Extractable color}

Extractable color was determined following ASTA 20.1, which is widely used by the spice industry for evaluation of color [18]. 0.1 g was weighed and placed in an amber colored $100 \mathrm{ml}$ volumetric flask and the volume was made up with acetone. The samples were left in the dark at room temperature for $16 \mathrm{~h}$ for color extraction and absorbance was measured using a spectrophotometer (Shimadzu Spectrophotometer UV - 1800, Kyoto, Japan, at $460 \mathrm{~nm}$ wavelength using the solvent 
acetone as blank. The results were reported in ASTA units following the procedure. The samples were analyzed before and during storage at regular time intervals as mentioned earlier. The average of duplicate readings was used for each sample in each condition at each time point.

ASTA Extractable Color $=$ Absorbance at $460 \mathrm{~nm} X 16.4$ / Sample weight (g)

\section{Surface color or visual color}

Surface color was determined using HunterLab (HunterLab LabScan XE, Hunter Associates Laboratory Inc., Reston, VA, www. hunterlab.com), which includes lightness and chroma saturation. The samples were measured before and during storage, similar to extractable color as mentioned earlier. The Hunter $\mathrm{L}, \mathrm{a}, \mathrm{b}$ color space is a 3 dimensional rectangular color space based on the opponent - colors theory [19]. For the $\mathrm{L}$ value which is " $\mathrm{L}$ "(Lightness) axis, 0 value is black and 100 is regarded as white.

Similarly for "a" (red-green) axis, positive values are red, negative values are green and 0 is neutral. And for " $b$ " (blue-yellow) axis, positive values are yellow, negative values are blue and 0 is neutral. Change in color over time was calculated as Delta $\mathrm{E}(\Delta \mathrm{E})$, where " $\Delta \mathrm{E}$ " was the total color difference and was used to compare any change in color over time. The average of duplicate readings was used for each sample in each condition at each time point.

$$
\Delta E=\sqrt{\left(L_{f}-L_{i}\right)^{2}+\left(a_{f}-a_{i}\right)^{2}+\left(b_{f}-b_{i}\right)^{2}}
$$

Where $L_{i}, a_{i}$ and $b_{i}$ were the initial (Day " 0 " values - before storage) hunter readings for $\mathrm{L}$, $\mathrm{a}$ and $\mathrm{b}$

$L_{f}, a_{f}$ and $b_{f}$ were the readings obtained each time the samples were analyzed.

Several other color parameters such as $\mathrm{L} \mathrm{X}$ a values for color intensity, hue angle $\left(\tan ^{-1} \mathrm{~b} / \mathrm{a}\right)$ and PACI (paprika color index ie. (1000 $\left.{ }^{*} \mathrm{a}^{*}\right) /\left(\mathrm{L}^{*}+\right.$ hue angle $\left.\mathrm{h}\right)$ were also calculated and used in In our study we used $\mathrm{L}$ and a values instead of $\mathrm{L}^{\star}$ and $\mathrm{a}^{*}$.

\section{Statistical analysis}

The elevated and room temperature storage data was analyzed per product category (paprika, chili pepper, chili powder) in Microsoft Excel using Welch's two sample t-test with unequal variances to analyze difference of the population means between week 0 and week 24 data, to determine significant differences at $95 \%$ confidence interval $(\mathrm{p}<0.05$, 2 -tailed). The data was further analyzed for calculating correlation coefficients (Pearson's $\mathrm{r}$ ) between various analytical parameters. Finally, the data was also analyzed to calculate rate constants for extractable color (ASTA) and Hunter values (L X a) for elevated and room temperature conditions, using first order reaction kinetics equation.

\section{Results and Discussion}

\section{Refrigerated and freezer conditions}

The results of our study indicated that there was minimal change observed in extractable color (ASTA) and Hunter L, a, b values in all the samples, when stored under refrigerated and freezer conditions for 6 months. Only paprika sample 6 (no ethoxyquin and high ASTA) showed $\sim 30 \%$ loss in extractable color in refrigerated conditions. All other samples had $<5 \%$ loss in extractable color and surface color (L $x$ a) in refrigerated and freezer storage conditions. Overall, there was a slight decrease in moisture content and no major change in water activity in all samples stored in refrigerated and freezer conditions. The relative humidity (\% RH) of refrigerator and freezer were $55 \%$ and $45 \%$ respectively and hence there was minimal absorption or desorption of water vapor in these conditions, resulting in stable moisture and water activity results.

Researchers suggest that a sharp change in the rate of color loss is observed at $15^{\circ} \mathrm{C}$ : the value $\mathrm{Q}_{10}\left({ }^{\circ} \mathrm{C}\right)$ changes from 1.62 to 2.82 when the temperature rises above $15^{\circ} \mathrm{C}$ [20]. Other researchers also suggest that evolution of carotenoid content indicated that autoxidative reactions are minimal and that coloring capacity is maintained when paprika samples are stored under controlled storage $\left(4^{\circ} \mathrm{C}\right.$ and $\left.70 \% \mathrm{RH}\right)$ [21]. Studies have shown that the enzyme extracted carotenoid pigments from orange peel, sweet potato and carrot samples were first freeze dried and stored at different temperatures. These samples suffered minimal pigment loss at $4^{\circ} \mathrm{C}$ and maximum loss was observed at $40^{\circ} \mathrm{C}$ and freeze drying lowered pigment loss for all samples under all conditions [22]. Hence, all of our results and discussion in this section will mainly focus on changes in analytical parameters in elevated and room temperature storage.

\section{Elevated and room temperature conditions}

In general, there was an increase in moisture and water activity in all samples upon storage in elevated conditions $\left(35^{\circ} \mathrm{C}, 80 \% \mathrm{RH}\right)$. At room temperature $\left(\sim 22^{\circ} \mathrm{C}, 45 \% \mathrm{RH}\right)$, all samples generally showed a decrease in moisture and water activity, mainly attributed to desorption of moisture from the products. Our main focus in the rest of the results section will be mainly on extractable color loss (ASTA) and surface color loss (Hunter).

Hunter color parameters $\mathrm{L}, \mathrm{a}$, and $\mathrm{b}$ values were used to calculate $\mathrm{L} \mathrm{X}$ a and Paprika Color Index (PACI) results which are referred in some of the results discussed in this section. Studies conducted on heating of different paprika samples for different times between $100^{\circ}$ $150^{\circ} \mathrm{C}$ suggest that the Hunter a value is related to carotenoid content decreasing with heat degradation of carotenoids; the decrease in L value is a function of the logarithm of percent increase in browning compounds [23]. It is also suggested that both $a$ and $L$ values decrease with time of heating (increasing changes of color), and hence the authors suggest that it may be appropriate to use a X L value to indicate color quality that provides an expanded scale. A new color index for paprika (PACI) is proposed based on the CIELAB coordinates $L^{*}$ (lightness), $\mathrm{a}^{\star}$ (red-blue), and $\mathrm{h}$ (hue angle), and it is calculated as " $1000 \mathrm{a}^{\star} /\left(\mathrm{L}^{\star}+\mathrm{h}\right)$ ". This new index shows a high correlation with the logarithm of extractable color $(\mathrm{r}=0.9662)$ and is able to distinguish between sample groups of different ASTA units [24]. In our study, we calculated PACI by using $L$ and a values instead of $L^{*}$ and $a^{*}$ values and compared correlations with Ln (ASTA) for different samples. We found that for elevated storage conditions, the average correlation coefficient (" $r$ ") between PACI and Ln (ASTA) values for the 7 paprika samples was 0.98 , for the 3 chili pepper samples was 0.99 and for the 2 chili powders was 0.99 , which were very similar to the values reported by other researchers.

\section{Welch's t-test for mean comparison at 0 and 24 weeks}

The Welch's two sample t-test results assuming unequal variances, 2 - tailed (Table 2) indicated that for the 7 paprika samples combined, there was significant difference $(p<0.05)$ between week 0 and week 24 data for extractable color (ASTA) and Hunter L X a values when data was analyzed combining observations for elevated and room 


\begin{tabular}{|c|c|c|c|c|c|c|}
\hline Sample Type & EL + RT & EL & RT & EL + RT & EL & RT \\
\hline Paprika & $*$ & $*$ & NS & $*$ & $*$ & NS \\
\hline Pepper & NS & NS & NS & NS & NS & NS \\
\hline Powder & $*$ & NS & NS & NS & NS & NS \\
\hline
\end{tabular}

$\mathrm{t}$-Test: Two-sample assuming unequal variances $\mathrm{p}<0.05$, Two-tail

*=significant, there are significant differences between week 0 and week 24 NS=non-significant, there are no significant differences between week 0 and 24

Where, $\mathrm{EL}+\mathrm{RT}=$ combined data from elevated and room temperature storage, $E L=e l e v a t e d$ storage

conditions only, RT=room temperature storage only

Table 2: Welch's two sample t-test data analysis for differences in mean values for ASTA and $L X$ a between week 0 and week 24 data.

temperature conditions and also for elevated conditions analyzed individually. There was no significant difference in ASTA and L X a value between week 0 and week 24 at room temperature storage. Researchers have shown that the color change of red pepper powder was greatly dependent on temperature and Aw and that as temperature and Aw increased, red color of pepper powder increasingly faded out to become brown and tarnish black, which is mainly attributed to the degradation of carotenoid pigments and development of browning compounds [25].

There was also no significant difference between week 0 and week 24 data for ASTA and L X a value for the 3 pepper samples combined, at elevated and room temperature storage. For the 2 powder samples combined, there was significant difference in week 0 and week 24 data when data was analyzed by combining observations in elevated and room temperature storage, but no significant difference when data in both conditions was analyzed separately. We believe a lot of the nonsignificance in the elevated conditions for the chili pepper and chili powder samples for ASTA and L X a has to do with the low sample size, attributing to low degrees of freedom (df).

\section{Correlation between analytical parameters}

The elevated and room temperature data was further analyzed to observe for correlation coefficients (Pearson's r) between various analytical treatments for paprika, chili peppers and chili powders (Tables 3a-3c). Across the 7 paprika, 3 chili pepper and 2 chili powder samples, combining all of the elevated and room temperature conditions data over 24 weeks, a significant positive correlation $(r>0.8)$ was noted between moisture and water activity. The Pearson $r$ values for paprika for correlation between moisture and water activity were 0.921 for paprika, 0.846 for chili pepper and 0.917 for chili powder samples. Also, a significant negative correlation $(\mathrm{r}<-0.8)$ was noted between ASTA and hue angle values. The Pearson $r$ values for these parameters were -0.947 for paprika, -0.982 for chili pepper and -0.945 for chili powder samples. These correlations are highlighted by ${ }^{*}$ in columns in the Table 3. Also, for individual sample categories, we noticed a few other significant $(r>0.8)$ positive and negative correlations.

\section{Rate constants calculations for elevated and room temperature conditions}

ASTA and L X a value data for elevated and room temperature storage conditions was analyzed to calculate rate constants of degradation using first-order kinetics, using the equation:

$$
\begin{aligned}
& \ln [\mathrm{Ct} / \mathrm{CO}]=-\mathrm{kt} \\
& \text { Where, } \mathrm{C}_{0}=\text { initial value of ASTA or L X a } \\
& \mathrm{C}_{\mathrm{t}}=\text { value of ASTA or L X a at time " } \mathrm{t} \text { " }
\end{aligned}
$$

\section{$\mathrm{t}=$ time in weeks \\ $\mathrm{k}=$ rate constant in week ${ }^{-1}$}

The k-values were calculated over 24 weeks storage at elevated and room temperature conditions and a ratio was calculated by dividing the $\mathrm{k}$-value under elevated conditions with the $\mathrm{k}$ value under room temperature conditions. These results are shown in Table 4.

Based on Table 4, higher ratios would indicate that the rate of degradation is high in elevated conditions and rate of degradation is low in room temperature conditions. Lower ratios would indicate that the rate of degradation is high in both storage conditions. Based on this analysis, we found the samples without ethoxyquin (paprika 2, paprika 6 and powder 2) to have low ratios for extractable color (ASTA) analysis, since the extractable color (ASTA) degradation is high in these samples even in room temperature storage. Also, powder 1 has a lower ratio, because this powder despite containing ethoxyquin had very low moisture content and it has been shown that ASTA degradation tends to be higher at lower moisture content. It has been shown that for ground paprika samples stored under ambient temperature $\left(19-24^{\circ} \mathrm{C}\right)$ and humidity (24-42\%) storage for 4 months, samples with 6 or $9 \%$ prestorage moisture content showed higher ASTA color loss compared to samples with 15 or $18 \%$ pre-storage moisture content [26]. Researchers have also further shown that a $3 \%$ increase in moisture content, caused

\begin{tabular}{|c|c|c|c|c|c|c|}
\hline \multicolumn{7}{|c|}{ Table 3a: Paprika } \\
\hline \multicolumn{7}{|c|}{ Paprika Samples } \\
\hline & ASTA & Moisture & Aw & $L X a$ & Delta E & Hue Angle \\
\hline ASTA & $x$ & -0.469 & -0.323 & 0.087 & -0.393 & $(-) 0.947$ * \\
\hline Moisture & & $\mathbf{x}$ & $0.921^{*}$ & -0.086 & 0.683 & 0.558 \\
\hline Aw & & & $\mathbf{x}$ & -0.925 & 0.730 & 0.423 \\
\hline$L X a$ & & & & $x$ & -0.683 & -0.230 \\
\hline Delta E & & & & & $x$ & 0.332 \\
\hline Hue Angle & & & & & & $x$ \\
\hline \multicolumn{7}{|c|}{ Table 3b: Chili Pepper } \\
\hline \multicolumn{7}{|c|}{ Chili Pepper Samples } \\
\hline & ASTA & Moisture & Aw & $L X a$ & Delta E & Hue Angle \\
\hline ASTA & $x$ & -0.825 & -0.569 & -0.436 & -0.634 & (-) 0.982 * \\
\hline Moisture & & $x$ & $0.846^{*}$ & -0.067 & 0.853 & 0.825 \\
\hline Aw & & & $\mathbf{x}$ & -0.0393 & 0.911 & 0.561 \\
\hline$L X a$ & & & & $x$ & -0.352 & 0.397 \\
\hline Delta E & & & & & $x$ & 0.678 \\
\hline Hue Angle & & & & & & $x$ \\
\hline \multicolumn{7}{|c|}{ Table 3c: Chili Powder } \\
\hline \multicolumn{7}{|c|}{ Chili Powder Samples } \\
\hline & ASTA & Moisture & Aw & $L X a$ & Delta E & Hue Angle \\
\hline ASTA & $x$ & -0.867 & -0.900 & 0.848 & -0.632 & $(-) 0.945$ * \\
\hline Moisture & & $x$ & 0.917 * & -0.993 & 0.469 & 0.844 \\
\hline Aw & & & $x$ & -0.890 & 0.665 & 0.791 \\
\hline$L X a$ & & & & $x$ & -0.397 & -0.852 \\
\hline Delta E & & & & & $x$ & 0.457 \\
\hline Hue Angle & & & & & & $\mathbf{x}$ \\
\hline
\end{tabular}
a decrease in hue angle with more clean red color and a decrease in $\mathrm{L}$ values, resulting in a darker color [27].

*indicates significant $(r>0.8)$ positive or negative correlation between parameters across all 3 product categories

Table 3: Correlation coefficients (Pearson $r$ ) between analytical parameters combining elevated and room temperature data for various product categories. 


\begin{tabular}{|c|c|c|c|c|c|c|}
\hline \multicolumn{7}{|c|}{ Rate constants for ASTA and $L X$ a values in elevated and room temperature conditions } \\
\hline & ASTA EL - $k$ & ASTA RT - $k$ & & L X a EL - k & L X a RT - k & \\
\hline Sample ID & value & value & Ratio & value & value & Ratio \\
\hline Paprika 1 & 0.0103 & 0.0031 & 3.31 & 0.0180 & 0.0009 & 19.77 \\
\hline Paprika 2-no ethoxyquin & 0.0209 & 0.0085 & 2.47 & 0.0232 & 0.0014 & 16.10 \\
\hline Paprika 3-irradiation & 0.0186 & 0.0044 & 4.19 & 0.0324 & 0.0035 & 9.18 \\
\hline Paprika 4 & 0.0100 & 0.0018 & 5.49 & 0.0187 & 0.0011 & 16.44 \\
\hline Paprika 5 & 0.0182 & 0.0049 & 3.75 & 0.0169 & -0.0003 & -65.63 \\
\hline Paprika 6-no ethoxyquin & 0.0307 & 0.0263 & 1.17 & 0.0225 & 0.0018 & 12.73 \\
\hline Paprika 7 & 0.0112 & 0.0038 & 2.96 & 0.0219 & 0.0015 & 15.05 \\
\hline Pepper 1 & 0.0144 & 0.0049 & 2.94 & 0.0162 & 0.0005 & 33.63 \\
\hline Pepper 2-irradiation & 0.0174 & 0.0036 & 4.79 & 0.0302 & 0.0034 & 8.98 \\
\hline Pepper 3 & 0.0136 & 0.0043 & 3.21 & 0.0217 & 0.0006 & 38.37 \\
\hline Powder 1 & 0.0399 & 0.0237 & 1.69 & 0.0119 & 0.0003 & 44.49 \\
\hline Powder 2-no ethoxyquin & 0.0356 & 0.0280 & 1.27 & 0.0165 & 0.0007 & 22.86 \\
\hline
\end{tabular}

Important k-value discussed in results section are highlighted in "bold font"

Table 4: Rate constants for ASTA and LX a value in elevated and room temperature conditions for different product types.

Furthermore, for the Hunter L $\mathrm{X}$ a value analysis, we found the irradiated samples (paprika 3 and pepper 2) to have lower ratios, because these samples would degrade at a higher rate in both elevated and room storage conditions. These particular findings are highlighted in "bold font" in the table.

The rate constants also allow us to predict the rate of degradation of extractable color (ASTA) values and Hunter L X a values in samples at room temperature compared to elevated conditions. The paprika samples for ASTA values, the samples containing ethoxyquin will degrade 3.94 times slower at room temperature than at elevated conditions, compared to 1.82 times slower at room temperature than at elevated conditions for non ethoxyquin containing samples. Researchers have shown that after 4 months storage at ambient temperature, the control treatment lost $63 \%$ of the initial extractable color, the d-tocopherol treated samples lost $32 \%$, and the ethoxyquin treated paprika sample lost $6 \%$ color from their original value. The pepper samples for ASTA values would degrade 3.65 times slower at room temperature than at elevated conditions and powder samples at 1.48 times slower at room temperature than at elevated conditions. This proves that added ethoxyquin provides significant color protection to paprika based products at elevated and room temperature storage, similar to data shared by other researchers. The effect of ethoxyquin on preservation of ASTA color in same sample (with same starting ASTA) but with and without ethoxyquin is shown in Figure 1. The figure clearly indicates that in the sample set containing no ethoxyquin (blue line - elevated or green line - room temperature) the loss of ASTA values are significantly higher than in the same sample set containing ethoxyquin (red line - elevated or purple line - room temperature).

In the case of Hunter L X a value, for paprika samples, the irradiated sample would degrade 9.18 times slower at room temperature than at elevated temperature. The non-irradiated samples would degrade 16.02 times slower at room temperature compared to elevated temperature. For pepper samples, for $\mathrm{L} \mathrm{X}$ a value, the irradiated sample would degrade at 8.98 time slower rate at room temperature than elevated temperature. The non-irradiated samples would degrade at 36 time slower rate at room temperature compared to elevated temperature. This proves that irradiation treatment negatively affects the surface color (Hunter values) and increases the rate of degradation of paprika based products and that irradiation treatment provides energy to activate the color degradation reaction at a faster rate in both elevated and room temperature storage conditions. Researchers

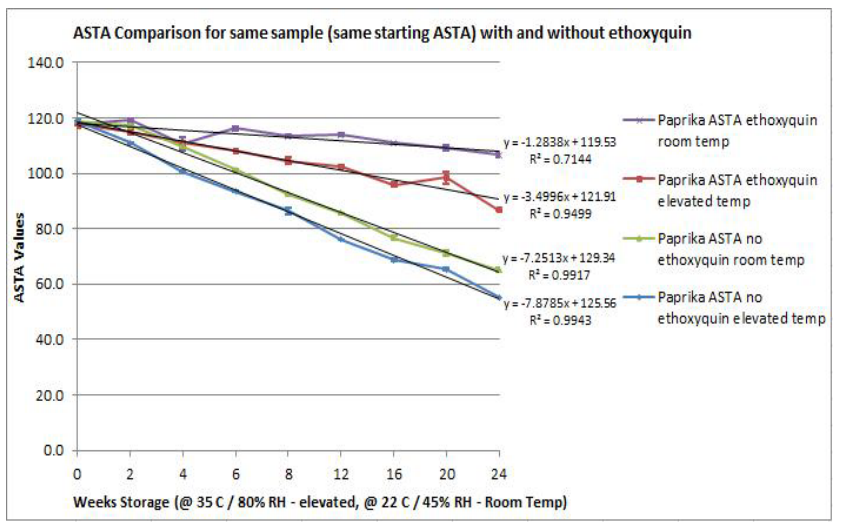

Figure 1: Extractable color (ASTA) comparison for same sample with and without ethoxyquin inelevated and room temperature conditions.

have similarly reported carotenoid degradation including capsanthin and other yellow pigments with irradiation treatment at $10 \mathrm{kGy}$ dose. Interestingly, irradiation treatment did not have a major effect on extractable (ASTA) color.

These ratios provide us with excellent information for future studies in this area and would help in saving considerable time conducting shelf life studies for new products for the industry. We could potentially conduct a quick shelf life study in elevated conditions and based on sample type, we could use these degradation rate constants to predict the shelf life at room temperature and other storage conditions, without having to complete the entire study at the lower storage temperatures.

\section{Conclusion}

This study shows that there was minimal extractable color (ASTA) and visual (Hunter) color loss in all 12 samples when stored at refrigerated and frozen conditions. There is a significant color loss for most samples for both color parameters when stored in elevated conditions. There was also a good correlation achieved between moisture and water activity and ASTA and hue angle measurements for all 12 samples under elevated and room temperature storage. Finally, there is a low rate constant ratio between elevated and room temperature conditions for ASTA measurements in samples not containing ethoxyquin, 
Citation: Addala R, Vasavada M, Dong J, Subramanian S (2015) Effect of Storage Conditions on Rate of Color Degradation of Paprika based Products. J Food Process Technol 6: 423. doi:10.4172/2157-7110.1000423

indicating a high loss of ASTA value even at room temperature conditions. There was also a low rate constant ratio between elevated and room temperature conditions for $\mathrm{L} \mathrm{X}$ a measurements in samples that had been subjected to irradiation treatment, indicating a higher loss $\mathrm{L} \mathrm{X}$ a values even at room temperature conditions.

\section{References}

1. Govindarajan VS, Rajalakshmi D, Chand N, Salzer UJ (1987) Capsicum Production, technology, chemistry, and quality. Part IV. Evaluation of quality, CRC Critical reviews in food science and nutrition, 25: 185-282.

2. Berke TG, Shieh SC (2012) Wood head Publishing series in food science, technology and nutrition.

3. Walker SJ (2011) Postharvest handling of dehydrated chiles. Guide H-236

4. Deli J, Molnar P, Matus Z, Toth G (2001) Carotenoid composition in the fruits of Red Paprika (Capsicum annuum var. lycopersiciforme rubrum) during ripening Biosynthesis of carotenoids in Red Paprika. J Agric Food Chem 49: 1517-1523.

5. De Guevara RGL, Gonzalez M, Garcia-Meseguer MJ, Nieto JM, Amo M, et al. (2002) Effect of adding natural antioxidants on color stability of paprika. Journal of science of food and agriculture 82: 1061-1069.

6. Lee DS, Chung SK, Kim HK, Yam KL (1991) Nonenzymatic browning in dried red pepper products. Journal of food quality 14: 153-163.

7. Priestley RJ, Effects of heating on foodstuffs. Applied science publishers limited, London.

8. Cremer DR, Eichner K (2000) Formation of volatile compounds during heating of spiced paprika (Capsicum annuum) powder. J Agric Food Chem 48: 24542460.

9. Lease JG, Lease EJ (1956a) Effect of fat soluble antioxidants on the stability of the red color of peppers. Food technology 10: 403-405.

10. Chen SL, Gutmanis F (1968) Auto-oxidation of extractable color pigments in chile pepper with special reference to ethoxyquin treatment. Journal of food science 33: 274-280.

11. Council of European Communities (1995) European Parliament and Council Directive of 20 February 1995 on food additives other than colors and sweeteners. Official journal of European communities.

12. FDA (1996) U.S. Food and Drug Administration
13. Nieto-Sandoval JM, Almela L, Fernandez-Lopez JA, Munoz JA (2000) Effect of electron beam irradiation on color and microbial bioburden of red paprika. $J$ Food Prot 5: 563-583.

14. Topuz A, Ozdemir F (2003) Influences of $Y$-Irradiation and storage on the carotenoids of sun-dried and dehydrated paprika. J Agric Food Chem 51: 4972 4977.

15. CFR - Code of Federal Regulations Title 21 (2014) Irradiation in the production, processing and handling of food.

16. ASTA method 2.1 (1997) Moisture in spices (Vacuum oven method).

17. ASTA method 6.0 (2010) Water activity

18. ASTA method 20.1 (1997) Spectrophotometric color assay - Oleoresin paprika and capsicum and ASTA method 20.1 - 1987 (Extractable color in capsicums and their oleoresins) from absorbance at $460 \mathrm{~nm}$. Hunter R S, Harold R W (1987) The measurement of appearance, John Wiley and Sons, Inc. New York, USA

19. Carbonell JV, Pinaga F, Yusa V, Pena JL (1986) The dehydration of paprika with ambient and heated air and the kinetics of color degradation during storage. Journal of food engineering 5: 179-193.

20. Perez-Galvez A, Hornero-Mendez D, Minguez-Mosquera MI (2009) Stability of paprika without supplementary antioxidants during storage under industrial controlled conditions. Journal of agricultural food chemistry 57: 4718-4723.

21. Cinar I (2003) Carotenoid pigment loss of freeze-dried plant samples under different storage conditions. LWT - Food science and technology 37: 363-367.

22. Ramakrishnan TV, Francis FJ (1973) Color and carotenoid changes in heated paprika. Journal of food science 38: 25-28.

23. Nieto-Sandoval JM, Fernandez-Lopez JA, Almela L, Munoz JA (1999) Dependence between apparent color and extractable color in paprika. Colo research and application 24: 93-97.

24. Rhim JW, Hong SI (2011) Effect of water activity and temperature on the color change of red pepper (Capsicum annuum L.) powder. Food science and biotechnology 20: 215-222.

25. Osuna-Garcia JA, Wall MM (1998) Pre-storage moisture content affects color loss of ground paprika (Capsicum annuum L.) under storage. Journal of food quality 21: 251-259.

26. Horvath $\mathrm{ZH}$, Hodur C (2007) Color of paprika powders with different moisture content. Institute of agrophysics 21:67-72.

27. Osuna-Garcia JA, Wall MM, Waddell CA (1997) Natural antioxidants fo preventing color loss in stored Paprika. Journal of Food Science 62: 1017 1021. 